\title{
HERBAL REMEDIES AND THEIR ADVERSE EFFECTS IN TEM TRIBE TRADITIONAL MEDICINE IN TOGO
}

\section{Tchadjobo Tchacondo ${ }^{1}$, Simplice D. Karou ${ }^{1 *}$, Komlan Batawila ${ }^{2}$, Amegninou Agban $^{1}$, Kawiwou Ouro-Bang'na ${ }^{1}$, Kokou T. Anani ${ }^{1}$, Mensavi Gbeassor ${ }^{1}$, Comlan de Souza ${ }^{1}$}

\author{
${ }^{1}$ Centre de Formation et de Recherche sur les Plantes Médicinales (CERFOPLAM), \\ Université de Lomé, ' 2 aboratoire de Botanique, Faculté de Sciences, Université de Lomé \\ *Ecole Supérieure des Techniques Biologiques et Alimentaires (ESTBA-UL), Université de \\ Lomé, BP 1515, Lomé, Togo \\ *Email: simplicekarou@hotmail.com
}

\begin{abstract}
In Africa, up to $80 \%$ of the population relies on herbal concoctions for their primarily health care. In Togo, western Africa, Tem tribe is a population with old knowledge of medicinal plants, however, still very little is known about their medical practices. The present study was conducted to access for the apprehension of adverse effects of traditional remedies by Tem traditional healers $(\mathrm{TH})$. Enquiry was performed by interviews with healers from August to October 2007 in Tchaoudjo prefecture (Togo). The study allowed us to interview $54 \mathrm{TH}$ including $41(75.93 \%)$ males and $13(24.07 \%)$ females, who cited 102 recipes assumed to have adverse effects. The recipes were used alone to cure several diseases including haemorrhoids $(22.55 \%)$, female sexual disorders and infertility $(21.57 \%)$, gastrointestinal disorders $(18.63 \%)$, and malaria $(6.86 \%)$. A total of 34 plants belonging to 21 families were cited to be components of the recipes. Euphorbiaceae and Mimosaceae families were the most represented, however, Nauclea latifolia, Khaya senegalensis, Pseudocedrela kotschyi and Xeroderris stuhlmannii were the main components of recipes linked to adverse effects. A total of 20 adverse effects were linked to the administration of theses drugs, and among them; diarrhoea, abdominal pains, polyuria, general weakness and vomiting were the most frequently encountered. These findings were in accordance with several reports of the literature concerning medicinal plants, although they were based on empirical observations. Laboratory screenings are needed to access for the effectiveness as well as the possible toxic effects of the recipes.
\end{abstract}

Keywords: traditional concoctions, medicinal plants, adverse effects, Togo.

\section{Introduction}

Plants continue to play a key role in health care systems in Africa, indeed up to $80 \%$ of people rely on traditional medicine (TM) for their primarily health care (WHO, 2009; Hostettman and Marston 2002). Because of this strong dependence on plants, a large number of studies have been conducted on traditional usage of plants and some often showed scientific rationale or resulted in the isolation of bioactive compounds for direct use in medicine (Castellanos et al., 2009; Karou et al., 2007a,b). However, still very little is known about African traditional medicine when compared to European and Asian systems (Ndhlala et al., 2009). The majority of works have been focussed on laboratory studies using plant extracts prepared by scientists after collecting plant materials themselves. The extractions are made with organic solvents such as chloroform, methanol or ethanol; sometimes they make decoctions in accordance with traditional practices (Akomo et al. 2009; Ouattara et al., 2007; Karou et al., 2006; Olila et al., 2001). The frequent scientific rational behind plants usage have stimulated a worldwide integration of TM with primary health care. However, the prescription and the use of traditional medicine in developing countries are not regulated, the main risk being the misadministration of toxic plants (Fennell et al., 2004). Nowadays, it is evidence that in developing countries people are using traditional concoctions, although very few works have been conducted on these vegetable drugs directly provided by traditional healers (TH); in fact the manufacturing of labelled and packaged herbal concoctions with claims to cure a wide range of diseases is still new in Africa. The recipes and preparations are of TM origin while the packaging and presentations are western; of course they still lack western safety and quality controls (Gertsch, 2009; Ndhlala et al., 2009).

Even modern drugs that pass safety and quality control tests have adverse effects; what about the traditional remedies? Plants commonly used in TM are assumed to be safe but this safety is based on their long usage in the treatment of diseases according to knowledge accumulated over centuries (Fennell et al., 2004). However, many studies demonstrated that some plants used as food or remedies are potentially toxic (Tchacondo et al., 2002, 2007; Maiga et al., 2005). As a consequence, some intoxication after consumption of plant remedies have been reported (Tchacondo et al., 2003a,b; Greenwald et al., 1996). The present study globally aimed to assess the apprehension of adverse effects of traditional remedies by TH. More especially we would like to: i) assess some recipes used to cure several diseases, ii) assess the main averse effects that may be linked to the administration of these products, iii) assess the scientific rationale of theses findings according to the literature. 


\section{Methodology Study area}

Central Region is one of the 5 administrative regions of Togo, a western African country. It lies between $0^{\circ} 15^{\prime}-1^{\circ} 35^{\prime}$ north and $8^{\circ}-9^{\circ} 15^{\prime}$ east. The region is made up of 4 prefectures : Tchaoudjo, Tchamaba, Sotouboua, Assoli (Figure 1), and is bordered to the north by Kara Region, to the west by Republic of Ghana, to the East by the Republic of Benin and to the south by Plateaux Region. It consists of a total area of 13,430 sq. $\mathrm{km}$ and occupies approximately $23.73 \%$ of the total $566000 \mathrm{sq}$. $\mathrm{km}$. land area of Togo mainland. The region belongs to tropical zone with one rainy season from April to October and one dry season from October to March. It receives 1200 to $1500 \mathrm{~mm}$ total rainfall annually. The annual temperatures are between 20 and $32^{\circ} \mathrm{C}$. The vegetation is essentially constituted of tree and bush savannah with excellent biodiversity of medicinal plants. The present study was carried out in Tchaoudjo prefecture. The prefecture is inhabited by 180,400 people, the main ethnic groups being the Tem people. Islam and animism are the main religions of the inhabitants; and agriculture and trade their principal activities. The prefecture has one Regional Hospital, 32 dispensaries and 3 pharmacies. For data collection, 54 healers were interviewed and the prefecture was divided into 5 districts: the centre with $68.52 \%$ $\mathrm{TH}$; the east, $14.82 \% \mathrm{TH}$; the west $3.70 \% \mathrm{TH}$; the north $11.11 \% \mathrm{TH}$ and the south $1.85 \% \mathrm{TH}$.

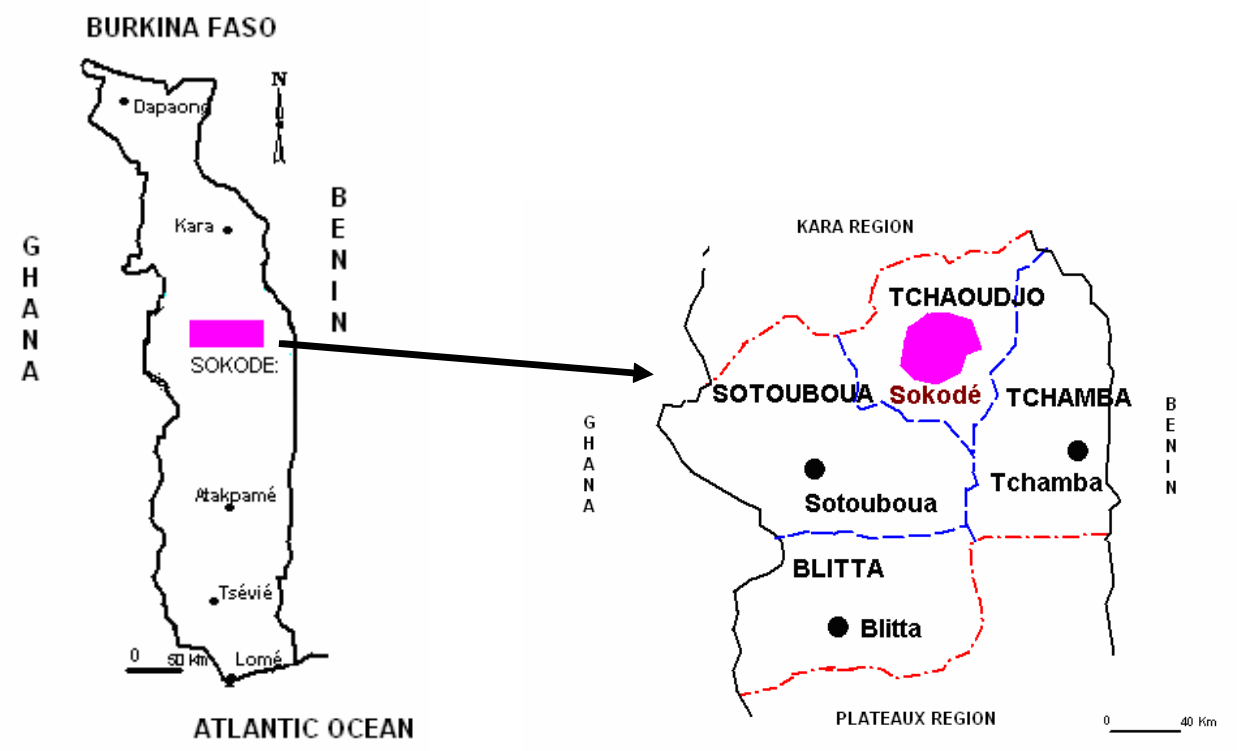

Figure 1: Map of the study area, geographic localisation of Togo and Togo Central region.

\section{Data collection}

Direct interviews with TH were performed between August and October 2007 using a semi structured questionnaire, after their informed consent. Each TH was asked to sign a consent form certifying his/her agreement with the form which was edited to explain the importance of the information they would provide. All TH were members of the Study and Research in Applied Traditional Medicine Centre of the Central Region in Togo (CERMETRA-RC). CERMETRA-RC is a non-governmental organisation created in August 2001. The organisation involves traditional healers of four prefectures of the Central Region in Togo. The goals of CEMETRA-RC are the formation and the counselling of $\mathrm{TH}$ about the management of patients and the preservation of the environment principally, the protection of vulnerable vegetable species used in traditional medicine. The organisation has a centre where TH follow up their patients and a botanic garden where they grow desired species. The organisation works in collaboration with researchers of University of Lomé. The interviewed TH were from Tchaoudjo prefecture (Figure 1). Questions asked were about the plants used in the preparation of remedies, the method of preparation of the remedies, the diseases the remedies are assumed to cure, the used parts of the plants, details of administration, the dosage and the adverse effects of their remedies. A remedy was included in the study if the $\mathrm{TH}$ himself recognised the presence of adverse effects after consumption of his product by patients. When any adverse effect was not shown by patients the products was not included in the study. 


\section{Plant identification}

After interviews, preliminary identification of the plants was done in the field by a botanist. Afterwards, herbarium specimens were prepared and photographs were taken to aid in the confirmation of the identity of the plants. Plant identities were confirmed by comparison with available voucher specimens in the Herbarium of the Botany Department of University of Lomé.

\section{Data analysis}

MS Excel spread sheets were used to make simple calculations and determine plant frequencies. The Informant consensus factor (ICF) was calculated for each category to identify the agreements of the informants on the reported cures for malaria symptoms. The ICF was calculated as follows: number of use citations in each category (Nuc) minus the number of species used (Ns), and divided by the numbers of use citations in each category minus one (Njoroge and Bussmann, 2007):

$$
I C F=\frac{N u c-N s}{N u c-1}
$$

Moreover, the fidelity level $(\mathrm{FI})$ was computed to determine the most important species used by the healers according to (Teklehaymnot, et al., 2007) as follows: frequency of citation of a species for a specific ailment (Fc) divided by the total number of citations of that species $(\mathrm{Ft})$.

$$
F l=\frac{F c}{F t} x 100
$$

\section{Results}

\section{Traditional healers}

In the present study, $54 \mathrm{TH}$ including $41(75.93 \%)$ males and $13(24.07 \%)$ females freely accepted to answer to our questionaire). Table 1 shows data on the age, the educational level and the occupation of the $\mathrm{TH}$. The ages could be divided in six age groups as indicated in the Table. The median age was 44 years and $60 \%$ of $\mathrm{TH}$ were between 30 and 50 years old. According to Table 1, the majority of the THs (48.16\%) were illiterates, $33.33 \%$ were of primary school level and $18.52 \%$ of secondary school level. Trade and agriculture were the main activities of the healers; however, the majority of the healers $(80.70 \%)$ were farmers.

Table 1: Ages, educational levels and occupations of interviewed Tem TH

\begin{tabular}{lllllll}
\hline Age groups & Illiterate & PSL & SSL & Farmers & Commercial & Total \\
\hline $\mathrm{X}<30$ & 2 & 0 & 4 & 6 & 0 & 6 \\
{$[30-39]$} & 2 & 12 & 6 & 17 & 3 & 20 \\
{$[40-49]$} & 9 & 6 & 0 & 13 & 2 & 15 \\
{$[50-59]$} & 4 & 0 & 0 & 4 & 0 & 4 \\
{$[60-69]$} & 5 & 0 & 0 & 5 & 0 & 5 \\
$X>70$ & 4 & 0 & 0 & 4 & 0 & 4 \\
\hline
\end{tabular}

PSL: primary school level, SSL: secondary school level

\section{Plants materials used in the concoction and formulations}

Different parts of the plants were used for the concoctions; these were roots, stems, leaves, barks, fruits (Figure 2). Sometimes the whole plant was used to prepare the recipes. All the portions could be used alone or in association with other parts. In our inquiry, roots (38\%) and stems $(22 \%)$ were the most frequently used. The recipes were essentially in 3 formulations including powders (48\%), decoctions (39\%) and maceration (13\%). 


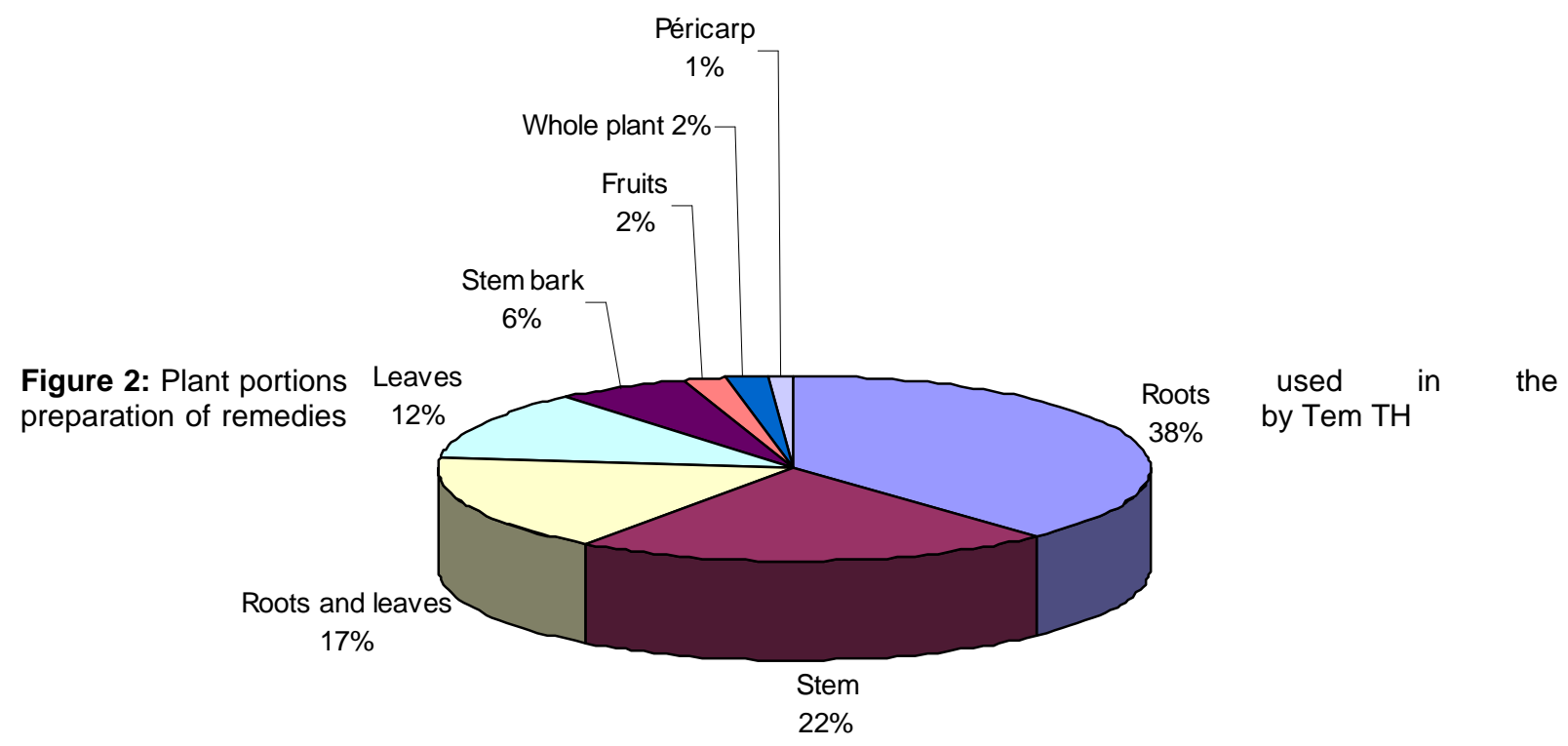

Plants, recipes and therapeutic indications

A total of 102 recipes assumed to have adverse effects were cited by healers. The recipes were used alone to cure several diseases including haemorrhoids (22.55\%), female sexual disorders and infertility $(21.57 \%)$, gastrointestinal disorders (18.63\%), and malaria (6.86\%) as indicated in Table 2. Each recipe could be made of a single plant or an association of 2 to 7 different plants at least. Overall, 34 plants were cited to be components of the recipes. The 34 plants belonged to 21 families as indicated in Table 3 which lists the plants and their local names. Euphorbiaceae and Mimosaceae families were the most represented with 4 species each. Melliaceae and Fabaceae were represented by 3 species each. The other families were represented by one or two species.

Table 2: Therapeutic indications of recipes provided by Tem TH

\begin{tabular}{ll}
\hline Diseases & $\begin{array}{l}\text { Percentage of Recipes } \\
(\mathrm{n}=102)\end{array}$ \\
\hline Haemorrhoids & 22.55 \\
Female sexual disorders and infertility & 21.57 \\
Gastrointestinal disorders & 15.83 \\
Malaria & 6.86 \\
Hepatitis & 4.90 \\
Hernia & 3.92 \\
Diabetes & 2.94 \\
Snakebites & 2.94 \\
Abscess & 2.94 \\
Sickle cells & 1.96 \\
Chicken-pox & 1.96 \\
Others & 8.83 \\
\hline
\end{tabular}

Data are the percentages of recipes proposed to cure each disease within the 102 recorded drugs. Diseases with recipes below $1 \%$ were designated as others.

\section{Number of citations and informant consensus factor.}

Data collected with healers revealed that a plant species could be used in the treatment of many diseases. According to Table 3, Nauclea latifolia (27 citations), Khaya senegalensis (26 citations), Pseudocedrela kotschyi (14 citations) and Xeroderris stuhlmannii (13 citations) were the most used plants. Thus these plants had low fidelity levels because of their multiple usages. Plants that were cited once were used to treat only one disease such as Lannea kerstingii, Kigelia africana or Maytenus senegalensis had very high fidelity levels $(100 \%)$. High consensus factors were recorded for haemorrhoids $(0.75)$, female sexual disorders and infertility $(0.63)$ and malaria (0.61). The lowest informant consensus was recorded with gastrointestinal disorders $(0.45)$. 
Table 3: Plants used in concoction of recipes with adverse effects by $\mathrm{Tem} \mathrm{TH}$, fidelity levels and total number of citation

\begin{tabular}{|c|c|c|c|c|c|c|c|}
\hline \multirow[t]{2}{*}{ Families } & \multirow[t]{2}{*}{ Species } & \multirow{2}{*}{$\begin{array}{l}\text { Vernacular } \\
\text { names }\end{array}$} & \multicolumn{4}{|c|}{ Fidelity levels } & \multirow[t]{2}{*}{$\mathrm{T}$} \\
\hline & & & $\mathrm{H}$ & FSDI & GID & $\mathrm{M}$ & \\
\hline Anacardiaceae & Lannea kerstingii Engl. \& K.Krause & Kelo & & 100 & & & 2 \\
\hline Aristolochiaceae & Aristolochia albida Duch. & Acadeyo & & & 100 & & 3 \\
\hline Bignoniaceae & Kigelia africana (Lam.) Benth. & Abliou & 100 & & & & 1 \\
\hline \multirow[t]{2}{*}{ Ceasalpiniaceae } & Cassia occidentalis L. & Kitchemtchem & & & 33.33 & 66.67 & 3 \\
\hline & $\begin{array}{l}\text { Piliostigma thonningii (Schum.) Milne- } \\
\text { Redh }\end{array}$ & Baco & & & 100 & & 1 \\
\hline Celastraceae & Maytenus senegalensis (Lam.) Exell & Tchichigan & & 100 & & & 1 \\
\hline Chrysobalanaceae & Parinari curatelifolia Planch. ex Benth. & Millimilou & & 40 & 40 & 20 & 5 \\
\hline Cochlospermaceae & $\begin{array}{l}\text { Cochlospermum planchonii Hook. f. } \\
\text { ex Planch }\end{array}$ & Koulobokou & & & & 100 & 1 \\
\hline \multirow[t]{2}{*}{ Combretaceae } & Pteleopsis suberosa Engl. \& Diels & Sisinon & 33.33 & 33.33 & 33.33 & & 3 \\
\hline & Terminalia avicennioides Guill. \& Perr & Souwo & & 33.33 & & 66.67 & 3 \\
\hline \multirow[t]{4}{*}{ Euphorbiaceae } & Bridelia ferruginea Benth. & Kolou & & 50 & 24 & 25 & 4 \\
\hline & Phyllanthus amarus Schum \& Thonn. & Seniseniyo & & & 100 & & 1 \\
\hline & Sapium grahanii (Stapf) Prain & Kachikadou & & & 100 & & 1 \\
\hline & $\begin{array}{l}\text { Securinega virosa (Roxb. ex Willd.) } \\
\text { Bail }\end{array}$ & Tchacatchaca & 33.33 & 33.33 & 33.33 & & 3 \\
\hline \multirow[t]{3}{*}{ Fabaceae } & $\begin{array}{l}\text { Millettia thonningii (Schum. \& Thonn.) } \\
\text { Bak. }\end{array}$ & Kodoliya & 33.33 & 66.67 & & & 9 \\
\hline & Pterocarpus erinaceus Poir. & Tem & 28.57 & 57.14 & 14.29 & & 7 \\
\hline & $\begin{array}{l}\text { Xeroderris stuhlmannii (Taub) } \\
\text { Mendonca }\end{array}$ & Tchalawari & & 84.63 & & 15.38 & 13 \\
\hline \multirow[t]{2}{*}{ Loganiaceae } & Anthocleista djalonensis A. Chev. & Assoubobissaou & 20 & 80 & & & 5 \\
\hline & Strychnos spinosa Lam. & Kpogbovourou & & 25 & 75 & & 4 \\
\hline \multirow[t]{3}{*}{ Meliaceae } & Khaya senegalensis (Desr.) A. Juss & Frimou & 53.85 & 30.77 & 15.38 & & 26 \\
\hline & $\begin{array}{l}\text { Pseudocedrela kotschyi (Schweinf.) } \\
\text { Harms }\end{array}$ & Ditoré & 35.71 & 7.14 & 28.57 & 35.71 & 14 \\
\hline & Trichilia emetica Vahl & Adjédjébgizou & 44.44 & 11.11 & 11.11 & 33.33 & 9 \\
\hline \multirow[t]{4}{*}{ Mimosaceae } & Entada africana Guill. et Perr. & N'doulou & & & 50 & 50 & 2 \\
\hline & Parkia biglobosa (Jacq.) Benth & Soulou & 100 & & & & 2 \\
\hline & $\begin{array}{l}\text { Pericopsis laxiflora (Benth ex Bak) } \\
\text { Van Meeuwen }\end{array}$ & tchemary & 83.33 & & 16.67 & & 6 \\
\hline & $\begin{array}{l}\text { Prosopis africana (Guill. et Perr.) } \\
\text { Taub }\end{array}$ & Kpalou & 14.29 & 14.29 & 14.29 & 57.14 & 7 \\
\hline Ochnaceae & Lophira lanceolata Van Tiegh ex Keay & Kparakpara & 66.67 & & 33.33 & & 3 \\
\hline Polygolaceae & Securidaca longepedunculata Fres. & Fozi & & & 50 & 50 & 2 \\
\hline Proteaceae & Protea madiensis Oliv. & Doudouride & & & 100 & & 1 \\
\hline Rubiaceae & Nauclea latifolia Smith & Kidjidjilou & 48.15 & 3.70 & 25.93 & 22.22 & 27 \\
\hline Rutaceae & Citrus aurantifolia L. & Akanka & & & 100 & & 1 \\
\hline Sapotaceae & Vitellaria paradoxa Gaertn C.F & Somou & 44.44 & 33.33 & 22.22 & & 9 \\
\hline Tiliaceae & Grewia venusta Fresen. & Bolo & 50 & 50 & & & 2 \\
\hline Vitaceae & Cissus aralioides & Bodi & & 100 & & & 1 \\
\hline
\end{tabular}

Fidelity level: H; haemorrhoids, FSD; female sexual disorders and infertility, GID; gastrointestinal disorders, M; malaria

$\mathrm{T}$ : total number of citations of species

\section{Adverse effects of recipes}

In the present study, the interviewed TH freely accepted that some of recipes they use to manage diseases possess adverse effects. This was based on patients' complaints. According to our results, haemorrhoids, female sexual disorders and infertility, gastrointestinal disorders and malaria were the most cited diseases cured by the recipes, thus the study was focussed on the adverse effects of the recipes used in the treatment of these diseases.

Haemorrhoids: A total of 23 recipes assumed to cure haemorrhoids were found to have adverse effect. All recipes were administrated by oral route except $\mathrm{PH}-23$ which was administrated via rectum in addition to oral 
route. These recipes were prepared from 17 plants according to Table 4 . Single plants (Trichilia emetica or Khaya senegalensis) or an association of 2 to 5 plants at least were used to cure haemorrhoids. All the parts of the plants were used in the preparation of the recipes but the roots of plants were the most frequently used parts. The recipes were essentially powders or decoctions. Khaya senegalensis, Nauclea latifolia, Pseudocedrela kotschyi, Trichilia emetica and Vitellaria paradoxa were the major plants used to cure haemorrhoids by being components of $17,12,6,5$ and 5 of the 23 recipes respectively (Table 4). According to the THs several adverse effects were linked to the administration of their drugs. In the case of haemorrhoids, 17 adverse effects were listed. Among them, diarrhoea polyuria and general weakness accounted for $69.57 \%$ (16/23), 60.87\% (14/23) and $56.52 \%$ $(13 / 23)$ remedies, respectively.

Table 4: Recipes used by Tem TH to cure haemorrhoids and their adverse effects

\begin{tabular}{|c|c|c|c|c|c|}
\hline Recipes & Plants & $\begin{array}{l}\text { Used } \\
\text { parts }\end{array}$ & Formulations & Administration & Adverse effects \\
\hline $\mathrm{PH}-1$ & Trichilia emetica & Roots & Powder & Oral & $\begin{array}{l}\text { Diarrhoea, general } \\
\text { weakness, abdominal pains, } \\
\text { vomiting }\end{array}$ \\
\hline $\mathrm{PH}-2$ & Trichilia emetica & Leaves & Powder & Oral & $\begin{array}{l}\text { General weakness, vomiting } \\
\text { headache }\end{array}$ \\
\hline $\mathrm{PH}-3$ & Khaya senegalensis & Stem bark & Powder & Oral & Diarrhoea, polyuria, hunger \\
\hline \multirow[t]{2}{*}{$\mathrm{PH}-4$} & Khaya senegalensis & Stem bark & Powder & Oral & $\begin{array}{l}\text { giddiness, sweating, } \\
\text { insomnia }\end{array}$ \\
\hline & $\begin{array}{l}\text { Securidaca } \\
\text { longepedunculata }\end{array}$ & Roots & & & \\
\hline \multirow[t]{3}{*}{$\mathrm{PH}-5$} & Khaya senegalensis & Stem bark & Powder & Oral & General weakness, nausea \\
\hline & $\begin{array}{l}\text { Securidaca } \\
\text { longepedunculata }\end{array}$ & Roots & & & \\
\hline & Nauclea latifolia & Roots & & & \\
\hline \multirow[t]{3}{*}{$\mathrm{PH}-6$} & Khaya senegalensis & Stem bark & Powder & Oral & General weakness, diarrhoea \\
\hline & Nauclea latifolia & Roots & & & \\
\hline & Grewia venusta & Roots & & & \\
\hline \multirow[t]{3}{*}{$\mathrm{PH}-7$} & Nauclea latifolia & Leaves & Decoction & Oral & $\begin{array}{l}\text { Diarrhoea, palpitations, } \\
\text { hunger }\end{array}$ \\
\hline & Millettia thonningii & Leaves & & & \\
\hline & Pterocarpus erinaceus & Leaves & & & \\
\hline \multirow[t]{3}{*}{$\mathrm{PH}-8$} & Khaya senegalensis & Stem bark & Powder & Oral & $\begin{array}{l}\text { Polyuria, general weakness, } \\
\text { hallucinations }\end{array}$ \\
\hline & Nauclea latifolia & Roots & & & \\
\hline & Pericopsis laxiflora & Roots & & & \\
\hline \multirow[t]{3}{*}{$\mathrm{PH}-9$} & Khaya senegalensis & Stem bark & Powder & Oral & $\begin{array}{l}\text { General weakness, } \\
\text { constipations }\end{array}$ \\
\hline & Nauclea latifolia & Roots & & & \\
\hline & Pericopsis laxiflora & Roots & & & \\
\hline \multirow[t]{3}{*}{$\mathrm{PH}-10$} & Khaya senegalensis & Leaves & Decoction & Oral & $\begin{array}{l}\text { Polyuria, general weakness } \\
\text { diarrhoea, insomnia }\end{array}$ \\
\hline & Nauclea latifolia & Leaves & & & \\
\hline & Pericopsis laxiflora & Leaves & & & \\
\hline
\end{tabular}




\begin{tabular}{|c|c|c|c|c|c|}
\hline \multirow[t]{3}{*}{$\mathrm{PH}-11$} & Nauclea latifolia & Roots & Powder & Oral & $\begin{array}{l}\text { Diarrhoea, polyuria, rectum } \\
\text { itching }\end{array}$ \\
\hline & Pterocarpus erinaceus & Roots & & & \\
\hline & Pericopsis laxiflora & Roots & & & \\
\hline \multirow[t]{3}{*}{$\mathrm{PH}-12$} & Nauclea latifolia & Roots & Powder & Oral & $\begin{array}{l}\text { Diarrhoea, general } \\
\text { weakness, polyuria. }\end{array}$ \\
\hline & Khaya senegalensis & Roots & & & \\
\hline & Pseudocedrela kotschyi & Roots & & & \\
\hline \multirow[t]{3}{*}{$\mathrm{PH}-13$} & Prosopis africana & Roots & Powder & Oral & Diarrhoea, general weakness \\
\hline & Parkia biglobosa & Roots & & & \\
\hline & Vitellaria paradoxa & Roots & & & \\
\hline \multirow[t]{3}{*}{$\mathrm{PH}-14$} & Khaya senegalensis & Stem bark & Powder & Oral & $\begin{array}{l}\text { general weakness, polyuria } \\
\text { abdominal pains }\end{array}$ \\
\hline & Nauclea latifolia & Roots & & & \\
\hline & Trichilia emetica & Roots & & & \\
\hline \multirow[t]{3}{*}{$\mathrm{PH}-15$} & Nauclea latifolia & Leaves & Decoction & Oral & $\begin{array}{l}\text { Diarrhoea, polyuria, } \\
\text { abdominal pains }\end{array}$ \\
\hline & Pseudocedrela kotschyi & Leaves & & & \\
\hline & Millettia thonningii & Leaves & & & \\
\hline \multirow[t]{3}{*}{$\mathrm{PH}-16$} & Khaya senegalensis & Stem bark & Decoction & Oral & $\begin{array}{l}\text { Polyuria, diarrhoea, } \\
\text { abdominal pains }\end{array}$ \\
\hline & Vitellaria paradoxa & Stem bark & & & \\
\hline & $\begin{array}{l}\text { Securidaca } \\
\text { longepedunculata }\end{array}$ & Roots & & & \\
\hline \multirow[t]{4}{*}{$\mathrm{PH}-17$} & Khaya senegalensis & Roots & Decoction & Oral & Polyuria, hunger \\
\hline & Anthocleista djalonensis & Roots & & & \\
\hline & Pseudocedrela kotschyi & Roots & & & \\
\hline & Pericopsis laxiflora & Roots & & & \\
\hline \multirow[t]{4}{*}{$\mathrm{PH}-18$} & Khaya senegalensis & Leaves & Decoction & Oral & $\begin{array}{l}\text { Polyuria, Diarrhoea, general } \\
\text { weakness, abdominal pains }\end{array}$ \\
\hline & Vitellaria paradoxa & Leaves & & & \\
\hline & Millettia thonningii & Leaves & & & \\
\hline & Pseudocedrela kotschyi & Leaves & & & \\
\hline \multirow[t]{3}{*}{$\mathrm{PH}-19$} & Khaya senegalensis & Stem bark & Powder & Oral & $\begin{array}{l}\text { Polyuria, diarrhoea, } \\
\text { abdominal pains }\end{array}$ \\
\hline & Nauclea latifolia & Roots & & & \\
\hline & Trichilia emetica & Roots & & & \\
\hline $\mathrm{PH}-20$ & Khaya senegalensis & Stem bark & Decoction & Oral & $\begin{array}{l}\text { Polyuria, diarrhoea, } \\
\text { giddiness }\end{array}$ \\
\hline
\end{tabular}




\begin{tabular}{|c|c|c|c|c|c|}
\hline & Lophira lanceolata & Roots & & & \\
\hline & $\begin{array}{l}\text { Securidaca } \\
\text { longepedunculata }\end{array}$ & Roots & & & \\
\hline & Pteleopsis suberosa & Roots & & & \\
\hline $\mathrm{PH}-21$ & Khaya senegalensis & Stem bark & Decoction & Oral & $\begin{array}{l}\text { Diarrhoea, abdominal pains, } \\
\text { hunger }\end{array}$ \\
\hline & Anthocleista djalonensis & Stem bark & & & \\
\hline & Vitellaria paradoxa & Stem bark & & & \\
\hline & Parkia biglobosa & Stem bark & & & \\
\hline $\mathrm{PH}-22$ & $\begin{array}{l}\text { Khaya senegalensis } \\
\text { Nauclea latifolia }\end{array}$ & $\begin{array}{l}\text { Stem bark } \\
\text { Roots }\end{array}$ & Decoction & Oral & $\begin{array}{l}\text { Polyuria Diarrhoea, general } \\
\text { weakness, allergies, sexual } \\
\text { disorders, abdominal pains, } \\
\text { sweating }\end{array}$ \\
\hline & Vitellaria paradoxa & Stem bark & & & \\
\hline & Securinega virosa & Roots & & & \\
\hline & Kigelia africana & Roots & & & \\
\hline $\mathrm{PH}-23$ & Khaya senegalensis & Stem bark & Decoction & Oral + rectal & $\begin{array}{l}\text { Polyuria, Diarrhoea, general } \\
\text { weakness }\end{array}$ \\
\hline & Nauclea latifolia & Roots & & & \\
\hline & Anthocleista djalonensis & Roots & & & \\
\hline & Lophira lanceolata & Roots & & & \\
\hline & Pseudocedrela kotschyi & Roots & & & \\
\hline
\end{tabular}

Female sexual disorders and infertility: The interviewed THs cited 22 recipes based on 20 plants for the treatment of female sexual disorders and infertility (Table 5). As for haemorrhoids, the recipes could be made of single plants or a combination of 2 to 5 different plants. Xeroderris stuhlmannii, Vitellaria paradoxa and Anthocleista djalonensis were used alone to cure female sexual weakness. However, Xeroderris stuhlmannii (11/22), Khaya senegalensis $(8 / 22)$ and Milletica thonningii (6/22) were the most frequently cited in the concoction of the products. The adverse effects linked to these products were essentially general weakness and polyuria in 59.09\% (13/22) and 40.09\% (9/22) cases, respectively over the 13 adverse effects cited by TH (Table 4).

Table 5: Recipes used by Tem TH to cure female sexual disorders and infertility and their adverse effects

\begin{tabular}{|c|c|c|c|c|c|}
\hline Recipes & Plants & $\begin{array}{l}\text { Used } \\
\text { parts }\end{array}$ & Formulations & Administration & Adverse effects \\
\hline $\mathrm{P}_{\text {TMIF1 }}$ & Xeroderris stuhlmannii & Roots & Powder & Oral & Polyuria \\
\hline $\mathrm{P}_{\text {TMIF2 }}$ & Vitellaria paradoxa & Roots & Powder & Oral & Polyuria, diarrhoea \\
\hline $\mathrm{P}_{\text {TMIF3 }}$ & $\begin{array}{l}\text { Anthocleista } \\
\text { djalonensis }\end{array}$ & Roots & Powder & Oral & $\begin{array}{l}\text { Diarrhoea vomiting, abdominal } \\
\text { pains }\end{array}$ \\
\hline $\mathrm{P}_{\text {TMIF4 }}$ & $\begin{array}{l}\text { Anthocleista } \\
\text { djalonensis }\end{array}$ & Stem bark & Decoction & Oral & $\begin{array}{l}\text { Sleepiness, general weakness } \\
\text { palpitations }\end{array}$ \\
\hline \multirow[t]{2}{*}{$P_{\text {TMIF5 }}$} & Xeroderris stuhlmannii & Roots & $\begin{array}{l}\text { Decoction + } \\
\text { powder }\end{array}$ & Oral & $\begin{array}{l}\text { Vomiting, general weakness } \\
\text { abdominal pains, polyuria }\end{array}$ \\
\hline & Lannea kerstingii & Stem bark & & & \\
\hline
\end{tabular}


Tchacondo et al., Afr J Tradit Complement Altern Med. (2011) 8(1):45-60

\begin{tabular}{|c|c|c|c|c|c|}
\hline $\mathrm{PT}_{\mathrm{MIF6}}$ & $\begin{array}{l}\text { Anthocleista } \\
\text { djalonensis } \\
\text { Millettia thonningii }\end{array}$ & $\begin{array}{l}\text { Stem } \\
\text { Stem }\end{array}$ & Decoction & Oral & Insomnia, general weakness \\
\hline \multirow[t]{2}{*}{$\mathrm{P}_{\mathrm{TMIF} 7}$} & Xeroderris stuhlmannii & Roots & Powder & Oral & Polyuria, sexual disorders \\
\hline & Grewia venusta & Roots & & & \\
\hline \multirow[t]{2}{*}{$\mathrm{P}_{\mathrm{TMIF8}}$} & Xeroderris stuhlmannii & Roots & Powder & Oral & $\begin{array}{l}\text { Polyuria, diarrhoea, sexual } \\
\text { disorders }\end{array}$ \\
\hline & Millettia thonningii & Stem & & & \\
\hline \multirow[t]{2}{*}{$P_{\text {TMIF9 }}$} & Millettia thonningii & Roots & Decoction & Oral & Diarrhoea \\
\hline & Strychnos spinosa & Roots & & & \\
\hline \multirow[t]{3}{*}{$P_{\text {TMIF10 }}$} & Xeroderris stuhlmannii & Roots & Decoction & Oral & Polyuria, abdominal pains \\
\hline & Khaya Senegalensis & Stem bark & & & \\
\hline & Pterocarpus erinaceus & Roots & & & \\
\hline \multirow[t]{2}{*}{$\mathrm{P}_{\mathrm{TMIF} 11}$} & $\begin{array}{l}\text { Anthocleista } \\
\text { djalonensis }\end{array}$ & Stem bark & Decoction & Oral & $\begin{array}{l}\text { Nausea, vomiting diarrhoea, } \\
\text { abdominal pains }\end{array}$ \\
\hline & Khaya Senegalensis & Stem bark & & & \\
\hline \multirow[t]{2}{*}{$\mathrm{P}_{\text {TMIF12 }}$} & Xeroderris stuhlmannii & Roots & Powder & Oral & $\begin{array}{l}\text { General weakness, insomnia } \\
\text { diarrhoea, polyuria }\end{array}$ \\
\hline & Khaya Senegalensis & Stem bark & & & \\
\hline \multirow[t]{3}{*}{$\mathrm{P}_{\mathrm{TMIF} 13}$} & Khaya Senegalensis & Stem bark & Powder & Oral & $\begin{array}{l}\text { General weakness, allergies, } \\
\text { abdominal pains }\end{array}$ \\
\hline & Parinari curatelifolia & Roots & & & \\
\hline & Bridelia ferruginea & Roots & & & \\
\hline \multirow[t]{3}{*}{$\mathrm{P}_{\mathrm{TMIF} 14}$} & Khaya Senegalensis & Stem bark & Powder & Oral & General weakness, vomiting \\
\hline & Pterocarpus erinaceus & Roots & & & \\
\hline & Millettia thonningii & Stem bark & & & \\
\hline \multirow[t]{3}{*}{$\mathrm{P}_{\mathrm{TMIF} 15}$} & Xeroderris stuhlmannii & Roots & Decoction & Oral & $\begin{array}{l}\text { Polyuria, vomiting, general } \\
\text { weakness }\end{array}$ \\
\hline & Khaya Senegalensis & Stem bark & & & \\
\hline & Millettia thonningii & Stem bark & & & \\
\hline \multirow[t]{3}{*}{$\mathrm{P}_{\text {TMIF16 }}$} & Xeroderris stuhlmannii & Roots & Powder & Oral & $\begin{array}{l}\text { Insomnia, abdominal pains, } \\
\text { allergies }\end{array}$ \\
\hline & Lannea kerstingii & Stem & & & \\
\hline & Cissus aralioides & Roots & & & \\
\hline \multirow[t]{3}{*}{$\mathrm{P}_{\mathrm{TMIF} 17}$} & Millettia thonningii & Roots & Decoction & Oral & $\begin{array}{l}\text { General weakness, vomiting, } \\
\text { sleepiness }\end{array}$ \\
\hline & $\begin{array}{l}\text { Terminalia } \\
\text { avicennioides }\end{array}$ & Roots & & & \\
\hline & Khaya senegalensis & Stem bark & & & \\
\hline \multirow[t]{2}{*}{$\mathrm{P}_{\mathrm{TMIF} 18}$} & Xeroderris stuhlmannii & Roots & Decoction & Oral & General weakness, polyuria \\
\hline & Bridelia ferruginea & Stem & & & \\
\hline
\end{tabular}




\begin{tabular}{|c|c|c|c|c|c|}
\hline \multirow{3}{*}{$P_{\text {TMIF19 }}$} & Pterocarpus erinaceus & \multicolumn{4}{|l|}{ Stem bark } \\
\hline & Securinega virosa & Roots & Decoction & Oral & Polyuria, diarrhoea, insomnia \\
\hline & Maytenus senegalensis & Roots & & & \\
\hline \multirow{3}{*}{$\mathrm{P}_{\text {TMIF20 }}$} & Vitellaria paradoxa & Roots & & & \\
\hline & Nauclea latifolia & Roots & Decoction & Oral & Polyuria, diarrhoea, insomnia \\
\hline & Trichilia emetica & Roots & & & \\
\hline \multirow{3}{*}{$\mathrm{P}_{\mathrm{TMIF} 21}$} & $\begin{array}{l}\text { Pseudocedrela } \\
\text { kotschyi }\end{array}$ & Roots & & & \\
\hline & Xeroderris stuhlmannii & Stem & Decoction & Oral & Vomiting, palpitations, polyuria \\
\hline & Pterocarpus erinaceus & Stem & & & \\
\hline \multirow{6}{*}{$\mathrm{P}_{\text {TMIF22 }}$} & Prosopis africana & Stem & & & \\
\hline & Pteleopsis suberosa & Stem & & & \\
\hline & Khaya Senegalensis & Stem bark & Decoction & Oral & $\begin{array}{l}\text { Diarrhoea, vomiting, polyuria, } \\
\text { insomnia, abdominal pains }\end{array}$ \\
\hline & Vitellaria paradoxa & Roots & & & \\
\hline & Xeroderris stuhlmannii & Roots & & & \\
\hline & Parinari curatelifolia & Roots & & & \\
\hline
\end{tabular}

Gastrointestinal disorders: Table 6 lists the recipes used to treat gastrointestinal disorders and the adverse effects linked to the administration of these remedies. According to the Table, 19 recipes made of 23 plants were used to treat gastrointestinal disorders. Securinega virosa, Nauclea latifolia, pericopis laxiflora, Khaya senegalensis, Securidaca longepedunculata, Cassia occidentalis, Sapium grahanii and Prosopis africana were cited as single plants curing gastrointestinal disorders. However these plants were also used in combination with other plants to cure the disease. Overall Nauclea latifolia (6/19), Khaya senegalensis (5/19) and Pseudocedrela kotschyi (4/19) were the most frequently cited plant in the management of gastrointestinal disorders. Nine adverse effects were cited by $\mathrm{TH}$ after consumption of their products. Overall, diarrhoea $84.21 \%(16 / 19)$ and vomiting $42.05 \%(8 / 19)$ were the most encountered adverse effects in the treatment of gastrointestinal disorders (Table 6).

Table 6: Recipes used by Tem TH to cure gastrointestinal disorders and their adverse effects

\begin{tabular}{|c|c|c|c|c|c|}
\hline Recipes & Plants & Used parts & Formulations & administration & Adverse effects \\
\hline $\mathrm{P}_{\mathrm{MV} 1}$ & Securinega virosa & Roots & Decoction & Oral & $\begin{array}{l}\text { vomiting, polyuria, general } \\
\text { weakness, insomnia }\end{array}$ \\
\hline $\mathrm{P}_{\mathrm{MV} 2}$ & Nauclea latifolia & Roots & Powder & Oral & $\begin{array}{l}\text { Diarrhoea, general } \\
\text { weakness, hunger }\end{array}$ \\
\hline $\mathrm{P}_{\text {MV3 }}$ & Pericopsis laxiflora & Roots & Decoction & Oral & Diarrhoea \\
\hline $\mathrm{P}_{\mathrm{MV} 4}$ & Khaya senegalensis & Stem bark & Powder & Oral & $\begin{array}{l}\text { Diarrhoea, general } \\
\text { weakness, vomiting }\end{array}$ \\
\hline $\mathrm{P}_{\text {MV5 }}$ & $\begin{array}{l}\text { Securidaca } \\
\text { longepedunculata }\end{array}$ & Stem bark & Powder & Oral & $\begin{array}{l}\text { Diarrhoea, vomiting, } \\
\text { palpitations }\end{array}$ \\
\hline$P_{\text {MV6 }}$ & Cassia occidentalis & Leaves & Powder & Oral & Diarrhoea, vomiting \\
\hline $\mathrm{P}_{\mathrm{MV} 7}$ & Sapium grahanii & Roots & Powder & Oral & Diarrhoea \\
\hline $\mathrm{P}_{\mathrm{MV} 8}$ & Prosopis africana & Stem & Powder & Oral & Diarrhoea, vomiting \\
\hline
\end{tabular}


Tchacondo et al., Afr J Tradit Complement Altern Med. (2011) 8(1):45-60

\begin{tabular}{|c|c|c|c|c|c|}
\hline \multirow[t]{2}{*}{$P_{\text {Mvg }}$} & Parinari curatelifolia & Roots & Powder & Oral & Insomnia \\
\hline & Vitellaria paradoxa & Roots & & & \\
\hline \multirow[t]{2}{*}{$P_{\text {MV10 }}$} & Strychnos spinosa & Roots & Powder & Oral & $\begin{array}{l}\text { Diarrhoea, vomiting, sexual } \\
\text { disorders }\end{array}$ \\
\hline & Entada africana & Roots & & & \\
\hline \multirow[t]{2}{*}{$P_{\text {MV11 }}$} & Pterocarpus erinaceus & Stem bark & Decoction & Oral & Diarrhoea, insomnia \\
\hline & Pteleopsis suberosa & Stem bark & & & \\
\hline \multirow[t]{2}{*}{$P_{M V 12}$} & Nauclea latifolia & Roots & Powder & Oral & Diarrhoea, allergies \\
\hline & Strychnos spinosa & Roots & & & \\
\hline \multirow[t]{2}{*}{$P_{M V 13}$} & Phyllanthus amarus & $\begin{array}{l}\text { Whole } \\
\text { plants }\end{array}$ & Decoction & Oral & vomiting, palpitations \\
\hline & Citrus aurantifolia & Pericarp & & & \\
\hline \multirow[t]{3}{*}{$P_{\text {MV14 }}$} & Khaya senegalensis & Stem bark & Decoction & Oral & Diarrhoea, polyuria \\
\hline & Nauclea latifolia & Roots & & & \\
\hline & Pseudocedrela kotschyi & Leaves & & & \\
\hline \multirow[t]{3}{*}{$P_{\text {MV15 }}$} & Nauclea latifolia & Roots & Powder & Oral & $\begin{array}{l}\text { Diarrhoea, vomiting, } \\
\text { allergies }\end{array}$ \\
\hline & Khaya senegalensis & Stem bark & & & \\
\hline & Pseudocedrela kotschyi & Roots & & & \\
\hline \multirow[t]{3}{*}{$P_{\text {MV16 }}$} & Nauclea latifolia & Roots & Powder & Oral & Diarrhoea, polyuria \\
\hline & Khaya senegalensis & Stem bark & & & \\
\hline & Aristolochia albida & Roots & & & \\
\hline \multirow[t]{3}{*}{$P_{\text {MV17 }}$} & Nauclea latifolia & Roots & Powder & Oral & Diarrhoea \\
\hline & Strychnos spinosa & Roots & & & \\
\hline & Aristolochia albida & Roots & & & \\
\hline \multirow[t]{4}{*}{$P_{M V 18}$} & Nauclea latifolia & Roots & Powder & Oral & Diarrhoea, palpitations \\
\hline & Aristolachia albida & Roots & & & \\
\hline & Lophira lanceolata & Roots & & & \\
\hline & Pseudocedrela kotschyi & Roots & & & \\
\hline \multirow[t]{4}{*}{$P_{\text {MV19 }}$} & Parinari curatelifolia & Roots & Powder & Oral & $\begin{array}{l}\text { polyuria, diarrhoea, } \\
\text { palpitations }\end{array}$ \\
\hline & Piliostigma thonningii & Roots & & & \\
\hline & Protea madiensis & Roots & & & \\
\hline & Pseudocedrela kotschyi & Roots & & & \\
\hline
\end{tabular}




$\begin{array}{ll}\text { Vitellaria paradoxa } & \text { Roots } \\ \text { Bridelia ferruginea } & \text { Roots } \\ \text { Trichilia emetica } & \text { Roots }\end{array}$

Malaria: Seven recipes made of 12 plants assumed to cure malaria were linked to adverse effects. No single plant was cited to cure the disease. All the recipes were made of combination of 3 to 5 plants at least. The most frequently cited plants were Nauclea latifolia (5/7), Pseudocedrela kotschyi (5/7), Prosopis africana (4/7) and Trichilia emetica (4/7). Six adverse effects were linked to the administration of the products. Among them polyuria $(7 / 7)$ and diarrhoea (6/7) were the most cited (Table 7).

Table 7: Recipes used by Tem TH to cure malaria and their adverse effects

\begin{tabular}{|c|c|c|c|c|c|}
\hline Recipes & Plants & Used parts & Formulations & administration & Adverse effects \\
\hline \multirow[t]{4}{*}{$\mathrm{P}_{\mathrm{P} 1}$} & Nauclea latifolia & Roots & $\begin{array}{l}\text { Maceration } \\
\text { Powder }\end{array}$ & Oral & Polyuria, allergies \\
\hline & Entada africana & Stem & & & \\
\hline & Parinari curatelifolia & Roots & & & \\
\hline & Prosopis africana & Stem & & & \\
\hline \multirow[t]{5}{*}{$\mathrm{P}_{\mathrm{P} 2}$} & Nauclea latifolia & Roots & Powder & Oral & Polyuria, allergies, \\
\hline & $\begin{array}{l}\text { Securidaca } \\
\text { longepedunculata }\end{array}$ & Roots & & & abdominal pains \\
\hline & Terminalia avicennioides & Roots & & & \\
\hline & Pseudocedrela kotschyi & Roots & & & \\
\hline & Cassia occidentalis & Roots & & & \\
\hline \multirow[t]{4}{*}{$\mathrm{P}_{\mathrm{P} 3}$} & Terminalia avicennioides & Roots & Powder & Oral & $\begin{array}{l}\text { Polyuria, vomiting, } \\
\text { diarrhoea }\end{array}$ \\
\hline & Trichilia emetica & Roots & & & \\
\hline & Prosopis africana & Roots & & & \\
\hline & Xeroderris stuhlmannii & Roots & & & \\
\hline \multirow[t]{4}{*}{$\mathrm{P}_{\mathrm{P} 4}$} & Nauclea latifolia & Roots & Powder & Oral & $\begin{array}{l}\text { Polyuria, diarrhoea, } \\
\text { abdominal pains }\end{array}$ \\
\hline & Prosopis africana & Roots & & & \\
\hline & Trichilia emetica & Roots & & & \\
\hline & Pseudocedrela kotschyi & Roots & & & \\
\hline \multirow[t]{4}{*}{$\mathrm{P}_{\mathrm{P} 5}$} & Nauclea latifolia & Roots & Powder & Oral & Polyuria, diarrhoea \\
\hline & Trichilia emetica & Roots & & & \\
\hline & Pseudocedrela kotschyi & Roots & & & \\
\hline & Cassia occidentalis & Roots & & & \\
\hline $\mathrm{P}_{\mathrm{P} 6}$ & Nauclea latifolia & Roots+ leaves & $\begin{array}{l}\text { Decoction } \\
\text { Powder }\end{array}$ & Oral & $\begin{array}{l}\text { Polyuria, vomiting, } \\
\text { diarrhoea }\end{array}$ \\
\hline
\end{tabular}




\begin{tabular}{llll} 
& \multicolumn{2}{c}{ Pseudocedrela kotschyi } & Roots + leaves \\
Bridelia ferruginea & Roots+ leaves & \\
$\mathrm{P}_{\mathrm{P} 7}$ & Nauclea latifolia & Roots & Powder \\
$\begin{array}{l}\text { Cochlospermum } \\
\text { planchonii } \\
\text { Xeroderris stuhlmannii }\end{array}$ & Roots & Roots \\
\hline $\begin{array}{ll}\text { Prosopis Africana } \\
\text { Pseudocedrela kotschyi }\end{array}$ & Roots \\
\hline
\end{tabular}

\section{Discussion}

The present study aimed to assess traditional recipes used by Tem people to cure several diseases and that are assumed to possess adverse effects. The enquiry allowed us to interview 54 TH who provided us 102 recipes and the diseases they are assumed to cure.

All the products were plant-based remedies; no additional product was used in the concoction of the remedies. But additives such as honey or milk may be added to the preparation for oral administration, principally in the case of bitter remedies to improve taste. A peculiarity with Tem people, indigenous of Togo central region, is that, they have no traditional alcohol drink. Thus no alcohol is added in their preparations, in contrast with Togo southern people who have "Sodabi" distilled from palm wine and the northern people who have "Tchakpalo" and "Tchoukoutou" local beer prepared from sorghum. In addition, as they are mainly Muslims, many Tem TH claimed that they do not accompany their practices with animal sacrifices.

Haemorrhoids, female sexual disorders, infertility, gastrointestinal disorders and malaria were the most cited. These diseases accounted for $66.81 \%(71 / 102)$ of recipes. Haemorrhoid is a disease well known in TM and TH use numerous plants for its management. Cathode campanulas a and Ficus Exasperata in Cameroon (Focho et al., 2009), Achyranthes aspera, Allium cepa, Combretum racemosum and Monodora myristica in Nigeria (Ajibesin et al., 2008), Piliostigma thonningii and Cochlospermum tinctorium in Mali (Togola et al., 2005) Ziziphus spina-christi by Yemenite Jews (Dafni et al., 2005) are the mains examples. Considering the recipes proposed against haemorrhoids by Tem TH, Khaya senegalensis, Nauclea latifolia, Pseudocedrela kotschyi, Trichilia emetica and Vitellaria paradoxa were the most usually used. Among these plants, Trichilia emetica is of common usage with above mentioned plants, this suggests certain similarities in plants usage between Tem TH and other $\mathrm{TH}$.

Many plants have been used in Africa as remedies to regulate the menstrual cycle, enhance fertility and as either abortifacients or antiabortifacients (Steenkamp, 2003). According to our results, Xeroderris stuhlmannii, Khaya senegalensis and Milletica thonningii were the most cited as being a part of recipes proposed against female sexual disorders and infertility by Tem TH. There are very few reports on these plants in this topic; the literature only reports the use of Trichilia emetica in the treatment of such diseases. Thus, in eastern Africa, the root bark decoction is used to make women fecund and in Senegal the leaf decoction is used against blennorrhoea (Burkill, 1997), however these findings need to be confirmed by laboratory screenings. In contrast Nauclea latifolia which is not frequently used by Tem people in the treatment of female sexual disorders and infertility has shown a good in vitro estrogenic activity (Njamen et al., 2008). In addition, Anthocleista djalonensis which is used as single plant in the management of sexual disorders in the present study has been found to exert good antimicrobial activity against non-gonococcal urethritis bacteria (Okoli and Iroegbu, 2004).

The following plants: Nauclea latifolia, Khaya senegalensis and Pseudocedrela kotschyi most cited in the present study as being the components of recipes used in the treatment of stomach-ache or gastrointestinal disorders are well known for their activity against several pathogens responsible for gastroenteritis. Khaya senegalensis for example has a good antimicrobial activity related to its polyphenols contents (Karou et al., 2005). Nauclea latifolia was found to exert antibacterial, anthelminthic, Antiamoebic and spasmolytic activities in laboratory screenings (Onyeyili et al., 2001; Fakae et al., 2000; Tona et al., 2000). The antibacterial and antiparasitic activities of Pseudocedrela kotschyi are also well documented (Kassim et al., 2009; Asase et al., 2008; Ogundiya et al., 2006).

Malaria is the most important parasitic disease that causes major health problem in Africa. In Togo, the disease accounts for up to $15 \%$ hospitalisations. Indeed, THs have long experience in the management of the disease. Many Togolese medicinal plants have been screened for the in vitro antiplasmodial activity (Karou et al., 2003; Gbeassor et al., 1989). These screenings often show scientific rational behind plant usage in the management of malaria by Togolese TH. In the present study, Nauclea latifolia, Pseudocedrela kotschyi, Prosopis africana and Trichilia emetica were the most cited in the treatment of malaria. All these plants have been 
screened with success for their in vitro antimalarial activity. Among these plants, Nauclea latifolia is the best known antimalarial plant according to recorded results (Asase and Oppong-Mensah, 2009).

A total of 20 adverse effects linked to the administration of theses drugs were cited. Overall, five adverse effects were common to all these recipes; these were diarrhoea, abdominal pains, polyuria, general weakness and vomiting. Some studies reported that these were the main adverse effects often encountered after consuming vegetable drugs (Togola et al., 2005; Castot et al., 1997; Chan et al, 1994). Indeed, Langasou et al. (1994) reported that gastroenteritis was the main adverse effect linked to the oral administration of plant-based remedies. In addition Greenwald et al. (1996) reported that female with sexual disorders often presented abdominal pains, general weakness and vomiting after treatment with Chinese herbal remedies.

Our results indicated that 5 plants Khaya senegalensis, Nauclea latifolia, Pseudocedrela kotschyi, Trichilia emetica and Posopis africana were the main components of recipes linked to adverse effects. Some of these plants are already known in the literature for their adverse effects. The toxic effects of Khaya senegalensis, particularly the seeds of the plant were earlier reported (Kerharo and Adam, 1974). Maiga et al (2005) reported that diarrhoea and vomiting were the toxic effects of Trichilia emetica, however, these adverse effects could be expected according to literature, since the plant is used as an emetic and a purgative against poisoning in some regions particularly in eastern Africa (Malgras, 1992).

The healers often claim that the adverse effects are generally due to an overdose of the remedy however, the expected effect of the remedy may be diarrhoea, such as in the cases of constipation and intestinal worms. In addition, for intoxication treatment, the patient is supposed to eliminate the poisons by vomiting. According to the healers the adverse effects are generally moderate and should disappear at the end of the treatment. The commonly used remedy against diarrhoea and vomiting is a cold bath; another plant part can be used when the adverse effect is violent and stopping of the treatment is recommended.

The main difficulty of the study was that; sometimes, the same name was attributed to different plants by healers, or that gender (male or female) may be attributed to plants that were from different families. In these two cases, specimens were collected and examined with healers to have a consensus on the local name of the plants. This study showed that collaboration between scientists and $\mathrm{TH}$ is possible as they freely accepted to give us their recipes. This may be beneficial for scientists, TH and patients. The study also allowed us to assess the adverse effects linked to the administration of several traditional remedies. An important point of the study is the fact that TH freely accepted that their drugs may have adverse effects; however this is often based on empirical observations. In fact, certain adverse effects such as vomiting could be of nutritional origin or linked to the disease itself. In addition some patients often use modern drugs in association with traditional remedies so the adverse effects could be the results of the interactions of the two drugs. These are data we could not analyse because we had no access to them. We could not also assess the mortality linked to the administration of these remedies. Some of the recipes proposed by $\mathrm{TH}$ were in accordance with reports in literature showing similarities in plants usage between Tem TH and other TH in the rest of the world. The study also demonstrated the scientific rational behind plant usage by $\mathrm{TH}$ but most of recipes need to be investigated through pharmacological, toxicological and phytochemical studies to ascertain the effectiveness as well as the possible toxic effects.

\section{Acknowledgments}

The authors gratefully thank the staff of CERMETRA-RC and all the healers who took part in this study.

\section{References}

1. Ajibesin KK, Ekpoa BA, Bala DN, Essien EE, Adesanya SA (2008). Ethnobotanical survey of Akwa Ibom State of Nigeria. J. Ethnopharmacol. 11: 387-408.

2. Akomo OEF, Zongo C, Karou SD, Obame LC, Atteke C, Savadogo A, Traore AS (2009). In vitro antiplasmodial and antibacterial activities of Canthium multiflorum Schum and Thonn (Rubiaceae) extracts. Pak. J. Biol. Sci. 112: 919-923.

3. Asase A, Oppong-Mensah G (2009). Traditional antimalarial phytotherapy remedies in herbal markets in southern Ghana. J. Ethnopharmacol. In press

4. Asase A, Kokubun T, Grayer RJ, Kite G, Simmonds MS, Oteng-Yeboah AA, Odamtten GT (2008). Chemical constituents and antimicrobial activity of medicinal plants from Ghana: Cassia sieberiana, Haematostaphis barteri, Mitragyna inermis and Pseudocedrela kotschyi. Phytother Res. 22:1013-1016.

5. Burkill HM (1997). The Useful plants of West Tropical Africa. Vol 4. 2nd edition. Royal Botanic Gardens, Kew

6. Castellanos GRJ, Prieto JM, Heinrich M (2009). Red Lapacho (Tabebuia impetiginosa)-A global ethnopharmacological commodity? J. Ethnopharmacol. 121:1-13.

7. Castot A, Djezzar S, Deleau N, Guillot B, Efthymiou ML (1997). Une pharmaco vigilance hors des sentiers battus : La phytovigilance ou la pharmacovigilance des plantes médicinales. Thérapie 52 : $93-$ 154.

8. Chan TYK, Tomlison B, Tse LKK, Chan JCN, Chan WWM, Critchley J (1994). Aconitine Poisoning due to Chinese herbal medicines: A review. Viet. Hum. Toxicol. 36: $452-455$

9. Dafni A, Levy S, Lev E (2005). The ethnobotany of Christ's Thorn Jujube (Ziziphus spina-christi) in Israel. J.Ethnobiol. Ethnomed.5:17. 
10. Fakae BB, Campbell AM, Barrett J, Scott IM, Teesdale-Spittle PH, Liebau E, Brophy PM (2000). Inhibition of glutathione S-transferases (GSTs) from parasitic nematodes by extracts from traditional Nigerian medicinal plants. Phytother Res. 14:630-4.

11. Fennell CW, Lindsey KL, McGaw LJ, Sprag SG Stafford GI, Elgoraschi EE, Grace OM, van Staden J (2004). Assessing African medicinal plants for efficacy and safety: pharmacological screening and toxicity. J. Ethnopharmacol. 94: 205-217.

12. Focho DA, Newu MC, Anjah MG, Nwana FA, Ambo FB (2009). Ethnobotanical survey of trees in Fundong, Northwest Region, Cameroon. J.Ethnobiol. Ethnomed.5:17.

13. Gbeassor M, Kossou Y, Amégbo K, de Souza C, Koumaglo K (1989). Antimalarial effects of eight African medicinal plants. J. Ethnopharmacol. 25: $115-118$.

14. Gertsch J (2009). How scientific is the science in ethnopharmacology? Historical perspectives and epistemological problems. J. Ethnopharmacol. 122: 177-183.

15. Greenwald MS, Ko RJ, Loscutoff SM (1996). Lethal ingestion of some Chinese herba tea containing ch'an su. West J. Med. 164: 71-75.

16. Hostettman K, Marston A (2002). Twenty years of research into medicinal plants: results and perspectives. Phytochem. Rev. 1: 275-285.

17. Karou DS, Nadembega WM.C, Ilboudo PD, Ouermi D, Gbeassor M, De Souza C, Simpore J (2007a). Sida acuta Burm f: a medicinal plant with numerous potencies. Afr. J. Biotechnol. 6: 2953-2959

18. Karou D, Nadembega WMC, Ouattara L, Ilboudo PD, Canini A., Nikiéma JB, Simpore J, Colizzi V, Traore A.S (2007b). African ethnopharmacology and new drug discovery. Med. Plant. Sci. Biotechnol. 1: 61-69.

19. Karou D, Savadogo A, Canini A, Yameogo S, Montesano C, Simpore J, Colizzi V, Traore A.S (2006). Antibacterial activity of alkaloids from Sida acuta. Afr. J. Biotechnol. 8: 195-200

20. Karou D, Dicko MH, Simpore J, Traore AS (2005). Antioxidant and antibacterial activities of polyphenols from ethnomedicinal plants of Burkina Faso. Afr. J. Biotechnol. 4: 823-828

21. Karou D, Dicko MH, Sanon S, Simpore J, Traore SA (2003). Antimalarial activity of Sida acuta BURMF L. (Malvaceae) and Pterocarpus erinaceus POIR (Fabaceae) J. Ethnopharmacol. 89: 291-294.

22. Kassim OO, Loyevsky M, Amonoo H, Lashley L, Ako-Nai KA, Gordeuk VR (2009). Inhibition of in-vitro growth of Plasmodium falciparum by Pseudocedrela kotschyi extract alone and in combination with Fagara zanthoxyloides extract. Trans. R. Soc. Trop. Med. Hyg. 03: 698-702.

23. Kerharo J, Adam JG (1974). La Pharmacopée Sénégalaise Traditionnelle, Plantes Médicinales et Toxiques, ed., Vigot Frères. Paris. ISBN2-7114-0646-6.

24. Langasou RBF, Akunyili DN, Akubue PI (1994). A preliminary study of gastrointestinal effects of some Nigeria medicinal plants. Fitoterapia. 65: $235-240$.

25. Maiga A, Diallo D, Fane S, Sanogo R, Paulsen BS, Cisse B (2005). A survey of toxic plants on the market in the district of Bamako, Mali:traditional knowledge compared with a literature search of modern pharmacology and toxicology. J. Ethnopharmacol. 96:183-193.

26. Malgras D (1992). Arbres et arbustes guérisseurs des savanes maliennes. Edits. Karthala, Paris.

27. Ndhlala AR, Stafford GI, Finnie JF, Van Staden J (2009). In vitro pharmacological effects of manufactured herbal concoctions used in Kwa-Zulu-Natal South Africa. J. Ethnopharmacol. 122:117122.

28. Njamen D, Magne Ndé CB, Tanee Fomum Z, Vollmer G (2008). Effects of the extracts of some tropical medicinal plants on estrogen inducible yeast and Ishikawa screens, and on ovariectomized Wistar rats. Pharmazie. 63:164-8

29. Njoroge NG, Bussmann WR (2007). Ethnotherapeautic management of skin diseases among the Kikuyus of Central Kenya. Journal of Ethnopharmacology 111: 303-307.

30. Ogundiya MO, Okunade MB, Kolapo AL (2006). Antimicrobial Activities of some Nigerian Chewing Sticks. Ethnobot. Leaflets 10: 265-271.

31. Okoli AS, Iroegbu CU (2004). Evaluation of extracts of Anthocleista djalonensis, Nauclea latifolia and Uvaria afzalii for activity against bacterial isolates from cases of non-gonococcal urethritis. J. Ethnopharmacol. 92: 135-144.

32. Olila D, Opuda-Asibo J, Odyek O (2001). Bioassay-guided Studies on the Cytotoxic and in Vitro Trypanocidal Activities of a Sesquiterpene (Muzigadial) Derived from a Ugandan Medicinal Plant (Warburgia ugandensis). Afr Health Sci. 1(1): 12-15.

33. Onyeyili PA, Nwosu CO, Amin JD, Jibike JI (2001). Anthelmintic activity of crude aqueous extract of Nauclea latifolia stem bark against ovine nematodes. Fitoterapia. 72:12-21

34. Ouattara L, Koudou J, Obame LCE, Karou DS, Traore AS, Bessière JM (2007). Chemical composition and antibacterial activity of Cochlospermum planchoni Hook.f. ex Planch essential oil from Burkina Faso. Pak. J. Biol. Sci. 10: 4177-4179.

35. Steenkamp V (2003). Traditional herbal remedies used by South African women for gynaecological complaints. J Ethnopharmacol. 86:97-108

36. Tchacondo T, Ben Attia M, Rouefi F, Trabelsi H, Reinberg A, Bouzouita K, Boughattas NA, Sakly M (2002). Rythme circadien et neurotoxicité du nitroprussiate de sodium (NPS) chez la Souris. Influence de la privation alimentaire et de l'antidote thiosulfate de sodium. Rev F.S.B. 1: 118-129.

37. Tchacondo T, Moussa B, Boughattas A, Ben Attia M, Sakly M (2003a). Rythme circadien de la toxicité utérine du nitroprussiate de sodium. Rev. F.S.B. 2:104-111, 
38. Tchacondo T, Sani M, Ben Attia M, Rouafi F, Trabelsi H, Reinberg AK Bouzouita A, Sakly M, Boughattas NA (2003b). Variation circadienne de la neurotoxicité du nitroprussiate de sodium et de l'activité de la rhodanèse hépatique chez la Souris. In : Mécanismes et physiopathologie des fonctions rythmiques. Editions Vernazobrés - Grégo, Paris. pp: 397 - 405

39. Tchacondo T, Ben Attia M, Moussa B, Boughattas A, Sakly M (2007). Etude Comparative de la variation circadienne de la toxicité induite par le Nitroprussiate de Sodium (NPS) et le Cyanure de Potassium (KCN) chez la Souris. J. Sci.,7: 25 - 34.

40. Teklehaymanot T, Giday M, Midhin G, Mekonn Y (2007). Knowledge and use of medicinal plants by people around Debre Libanos monastery in Ethiopia. J. Ethnopharmacol 111: 271-283.

41. Togola A, Diallo D, Dembélé S, Barsett H, Paulsen BS (2005). Ethnopharmacological survey of different uses of seven medicinal plants from Mali, (West Africa) in the regions Doila, Kolokani and Siby. J. Ethnobiol. Ethnomed. 1:7.

42. Tona L, Kambu K, Ngimbi N, Mesia K, Penge O, Lusakibanza M, Cimanga K, De Bruyne T, Apers S, Totte J, Pieters L, Vlietinck AJ (2000). Antiamoebic and spasmolytic activities of extracts from some antidiarrhoeal traditional preparations used in Kinshasa, Congo. Phytomedicine. 7: 31-38.

43. WHO (2009). Traditional medicine. Available online from http://www.who.int/topics/traditional medicine len/ Accessed on October 2009

\section{Appendix 1: TH identity}

1. Date.....

2. Village.

3. Name and Surname.

4. Sex......

5. Ethnic group.

6. Religion.....

7. Address.

8. Educational level.

9. Origin of the knowledge.

10. Speciality.....

11. Adherence to CERMETRA-RC

\section{Appendix 2: Remedies}

1. Remedy Name

2. Number of plants in the remedy.

3. Local names of the plants.....

4. Scientific names.

5. Used parts.

6. Remedy formulation.

7. How do you prepare the remedy?

8. Which ailments the remedy is supposed to cure?.....

9. Administration route.

10. Dosage.

11. When do we take the remedy?.

12. Duration of the treatment.

13. Remedy storage.....

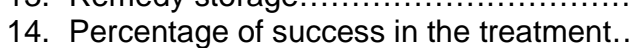

15. Interdictions.

16. Adverse effects.

17. Percentage of patients subject to adverse effects...

18. Delay of apparition of adverse effects.

19. Duration of the adverse effects.

20. How do you manage the adverse effects?

21. Other information. 